\begin{tabular}{|l|r|r|}
\hline Volume 3, Number 18 & Opinion & 31 JULY 2019 \\
\hline
\end{tabular}

\title{
Connected \& Semi-Autonomous Vehicles Can Save Lives Now!
}

By

Jim Mennie, Florida Polytechnic University

T

The state of California Department of Motor Vehicles has issued 60 permits to companies testing Autonomous Vehicles in the state as of October 2018 (California Dept. of MV, 2018). Billions of dollars are being invested by Waymo, GM, Tesla, Voyage, Ford, Mercedes, BMW, and Audi in the pursuit of the technology that will enable these companies to commercialize their products. While the investments are in the billions, it is estimated the A/V market will exceed $\$ 500$ billion within ten years. Depending upon who wins the race to bring the best $\mathrm{AV}$ product to market existing companies may not survive this technology dogfight. However, mass adoption of this technology is years away(Mennie 2019).

Meanwhile as this emerging technology is in the testing phase, Levels 1-3 of autonomous vehicles are in operation on our roads today. These autonomous features which include driver assist, emergency braking, highway driv- ing, lane assist, and parallel parking are providing a layer of safety in the market that has proven to be very popular with consumers. Additional safety features employed by connected vehicles and smart cities while also in testing phase have proved (Yue $\&$ Abdel-Aty, 2018) to reduce automobile accidents and improve safety. This technology exists today as several cities in the United States are currently utilizing in a pilot study.

Connected vehicles, unlike autonomous vehicles continuously receive information from a variety of sources providing real time traffic updates and safety information so that drivers can make informed decisions about where to go, how to avoid road hazards, and be alerted to changing traffic conditions. Additionally, pedestrians, cyclists, taxi drivers, and buses can also receive this information from either their smart phones, enhanced rear view mirrors (Serves as a monitor with messages and alerts), or an onboard screen.

Keywords: Connected Vehicles (CV's), Semi-Autonomous vehicles, Autonomous Vehicles technology, Smart Cities, DSRC

Copyright $\odot$ 2019, Jim Mennie. This article is published under a Creative Commons BY-NC license. Permission is granted to copy and distribute this article for non-commercial purposes, in both printed and electronic formats 
Autonomous Vehicles, when they come will be transformative, disruptive, and hopefully provide benefits that have been anticipated by its advocates. Expected productivity gains, reduction in highway fatalities and the added benefit of providing mobility to individuals who would not otherwise be mobile will help to improve American lives (Mennie, 2019). With the recent fatal crashes of $\mathrm{A} / \mathrm{V}$ test vehicles, the public's acceptance of this technology has waned as represented in several surveys recently conducted. Perception of safety is not matched by the actual record as the public may expect perfection which is just not achievable at this point. While we await the improvements and testing of this technology from a public safety perspective we may want to look at pursuing an avenue that has escaped focused attention. The safety benefits of Level 1-3 autonomous technology which is not fully autonomous and still has driver interface has proven to be very popular amongst consumers. But highway fatalities have approached almost 40,000 per year and this level of death is unacceptable. Why not combine the safety features of semi-autonomous vehicles with connected vehicles and give drivers a greater chance of survival and prevention of accidents?

The technology platforms of connected vehicles which are stand alone are currently being tested in several American cities and are not as complex as autonomous vehicle technology. The technology consists of radio antennas,

\section{Sixty percent of Americans know little to nothing about autonomous vehicles (Ramlet, 2018)}

not served to promote the safety of $\mathrm{A} / \mathrm{V}$ technology, thus increasing apprehension. Waymo, Google's autonomous vehicle division recently recorded its $10,000,000$ self-driven mile, plus billions of miles in computer simulation and possesses an enviable safety record unduplicated by human drivers. Traffic fatalities are expected to decrease $90 \%$ after mass adoption of Autonomous Vehicles (McKinsey \& Co., 2015), and connected vehicle technology is expected to reduce vehicle crashes between 15\%-70\% (Yue \& Abdel-Aty, 2018). If we extrapolate the expected improvements to vehicle safety utilizing the $90 \%$ figure when mass adoption of A/V technology is implemented, we can expect a reduction in fatalities of 36,000 per year. Annually, the U.S records 6 million car accidents, 3 million injuries in these accidents, and 2 million permanent injures (NHSTA, 2017). But we will have to endure another ten years of the full statistics until mass adoption occurs. If we rolled out connected vehicle technology with L1-L3 autonomous vehicle technology, we would not have to wait ten years to see significant reductions in automobile accidents injuries and fatalities.

\section{Expert perception}

a receiver, and a specially adapted rear view mirror that displays information in real time to the driver. This information can alert the connected vehicle driver to a wrong way driver, hazardous road conditions, traffic conditions, pedestrians in crosswalks, road hazards, and even safety reminders such as speed limits, stop signs and dangerous curves.

Combine this technology with lane assist, emergency braking, highway driving, parallel parking and you equip a driver with an arsenal of safety features the likes of which have never been seen. It may be enough to give drivers a fighting chance for increased survivability. Why would we not want that, and focus efforts on achieving this?

\section{The Perception}

Today, consumers are increasingly concerned about Autonomous Vehicles as they learn more about the technology. Unfortunately, in a consumer study last summer (Cox Automotive, 2018), 49\% of respondents stated they would not purchase a fully autonomous vehicle (Level 5), which was up from 30\% two years prior. The widely publicized recent accidents with Uber \& Tesla which resulted in fatalities have
In a recent interviews with Autonomous Vehicle experts respondent consensus indicated the positive benefits this technology would bring to society, as well as improve individual lives. Numerous companies are investing billions of dollars in this technology because their information shows the benefits, and the safety that will be delivered

\section{Public perception}

Public perceptions indicate a rising level of concern for this technology not only in surveys conducted earlier this year, but a rather fundamental lack of understanding of the technology. "Sixty percent of Americans know little to nothing about autonomous vehicles" (Ramlet, 2018). In order for the public to accept this technology, reduce or eliminate this misperception, an intensive education program needs to be implemented so the public can learn about this technology and their erroneous perceptions can be corrected.

\section{The Reality of the Present}

When considering AVs, there are many technologies involved. What they have in common is that all are either safer today than driving without them or are virtually certain to be safer once they are broadly adopted. 


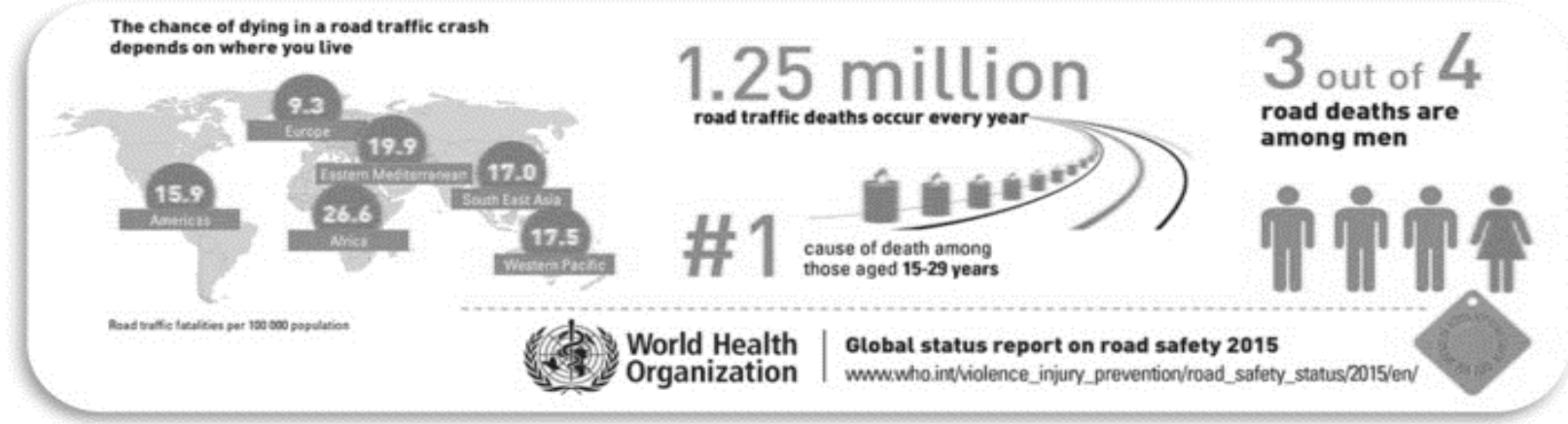

Figure 1: United Nations World Health Organization (2015)

Worldwide, over 1.25 million people die on the roads annually (see Figure 1), of which almost 40,000 are attributed to the U.S. This is an outrageous figure that we have passively accepted with no moral outrage. Where are the protests? Where are the marches? We protest tweets, and upholding existing laws, yet cannot muster the effort to demonstrate outrage and a call for action to significantly reduce this national and world-wide tragedy? If this many people died in terrorist attacks, or earthquakes would there be moral outrage? These deaths are preventable and Level 1-3 A/V technology and Connected Vehicle technology is here today.

Connected vehicle crash rates, dependent upon type of crash showed a reduction of between 15\%$70 \%$ (Yue \& Abdel-Aty 2018) in an analysis of crash data from 2005-2008. The safety features of L1-L3 vehicles (called semi-autonomous because they still require driver control) includes driver assist systems (DAS) such as emergency braking, lane assist, parking assist, highway driving. There are no safety statistics available evaluating the efficacy of L1-L3 levels of autonomy as compared to connected vehicles. However, all accidents have one commonality-human drivers. The advanced driver assistance features resident within L1-L3 autonomous vehicles can provide that additional layer of safety necessary to reduce accidents by replacing some of the functions performed by human drivers by technology. If we have an automobile perform some of the driving functions, should we expect increased safety?

Combining connected vehicle and semi-autonomous vehicles would further enhance safety for drivers and provide the best of both worlds, in essence doubling down and giving drivers a greater chance of survivability. There are obviously cost factors involved with added safety features and benefits. Connected vehicle technology equipment is less than $\$ 500.00$. Amortizing that cost over an 8-year auto life is less than $\$ 7.00$ a month. L1-L3 technology is significantly costlier. However, it is included in many car models today as standard equipment, while some of the features of L3 are optional at additional cost.

\section{Safety Implications of Emerging}

A variety of emerging technologies are expected to further increase the safety margin compared with how we drive today. Combining and converging the technology platforms that exist within semi-autonomous vehicles and connected vehicles today may help to significantly reduce the number of accidents and traffic fatalities by providing a plethora of safety features that will assist drivers in making better decisions and provide them with vital information so that better decisions can be made. These two technology platforms should not be stand alone. Why would a driver not want the best opportunities and utilization of the best technology to assist in safety?

\section{Mass Adoption of Autonomous}

A recent article on mass adoption of $\mathrm{A} / \mathrm{V}$ (Mennie, 2019) indicate this would not happen well into the 2020 's and beyond (Figure 2). As a society, we should not wait until this technology is perfected. As a society should we wait to receive the anticipated benefits of a 90\% reduction in fatalities (Ramsey, 2017)? Combining Level 1-3 Autonomous Vehicle technology, which is not fully automated and is available now and Connected Vehicle technology should be championed and incentivized. In conjunction with these Level 1-3 A/V technology, Connected Vehicle technology must be added to the equation as well so that we can provide drivers with as many safety features as possible and provide the requisite tools required to make the safest driving decisions. These issues should be elevated to a heightened focus of concern as it is unconscionable that we accept these horrific fatality figures EVERY YEAR.

\section{Connected Vehicles}

The city of Tampa, Florida has received a $\$ 17$ million grant from the U.S. Department of Transportation for its Connected Vehicle Pilot Deployment Program to test the validity and safety of this technology. Actual drivers volunteering for this program 


\section{Mass Adoption of Autonomous Vehicles}

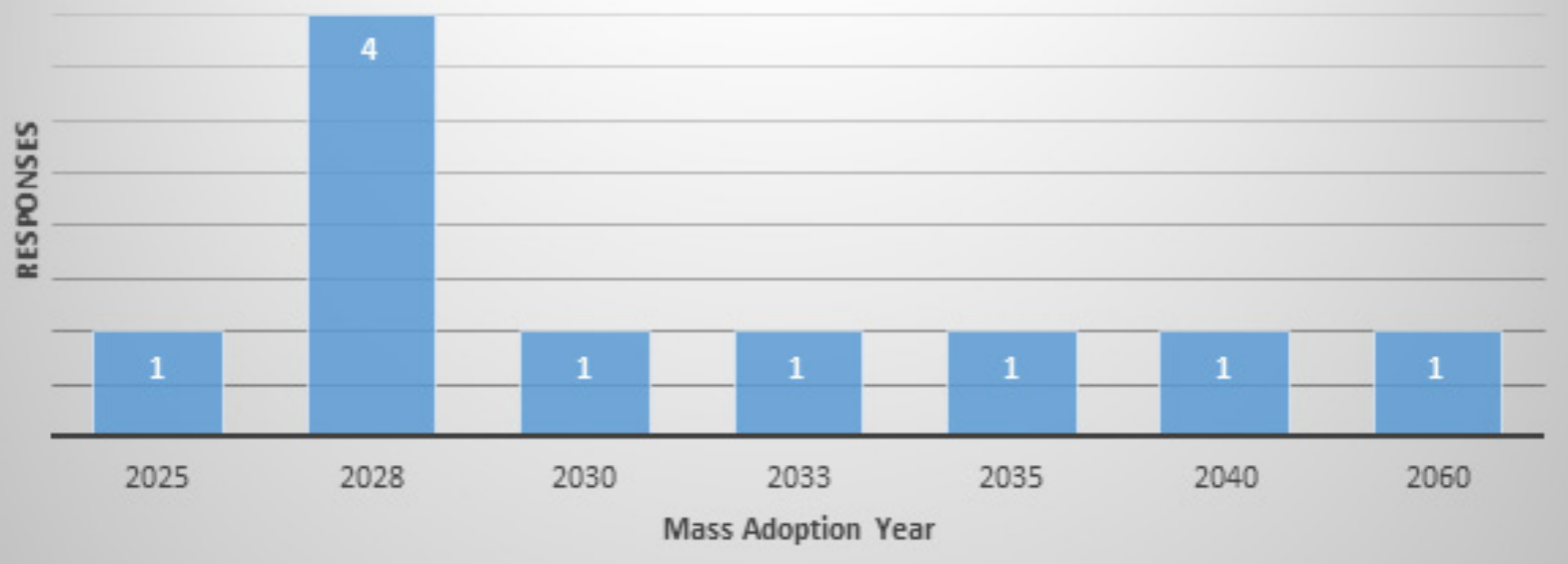

Figure 2: Mass Adoption of Autonomous Vehicles Source: (Mennie 2018 B)

receive free of charge all of the equipment necessary to participate in the program. The equipment consists of several antennas to transmit and receive information (central business district in downtown Tampa), a transceiver also known as dedicated short range communication device (DSRC), and an enhanced rear view mirror that replaces the drivers existing one. This new rear view mirror serves as a monitor providing real time information to the driver. The program has room for 1,600 privately owned vehicles, and as of October 2018 just under 1,000 drivers have signed up. Additionally, 10 buses and 10 streetcars in this area are also outfitted with this technology.

The city of Tampa has outfitted crosswalks, traffic signals and other infrastructure with 46 wireless communication sensors that communicate with all of the connected participants providing safety information in real time. Information is wirelessly communicated with connected participants to alert drivers when passengers enter a crosswalk. During congested traffic situations the information provided by the buses will enable traffic signals to modify their timing patterns to ease traffic congestion and assist the buses in maintaining their schedules. Even motorists and pedestrians uninvolved with the program will benefit with improved traffic management by The City of Tampa Traffic Management Center who will use real time data collected from sensors and participants to better manage traffic flow. There are anticipated positive environmental effects as when traffic flow is better managed, less idling and congestion will lead to a reduction in exhaust fume production, and increased fuel efficiency.

\section{Smart Cities}

Smart cities are those cities have made provisions to utilize technology to communicate with their citizens. Sensors and other data collection devices are located throughout the city and can provide information on parking, traffic, infrastructure and just about anything else. These sensors can be internet enabled and can communicate via cellular networks, and radio controlled environments. This information can be directly broadcast to users, or back to a server to be combined or streamlined with additional information and broadcast to users. Real time traffic information which includes road construction, accidents, hazardous conditions and alternate routes can be disseminated.

Connected vehicles can use the information provided by Smart Cities as well as other connected vehicles, infrastructure and pedestrians thus providing the most up to the minute traffic information. Smart Cities are not limited just to providing traffic information, but are capable of communicating and providing information on other matters such as areas in need of municipal services, water leaks, and police \& fire matters.

\section{Connected Semi-Autonomous Vehicles}

Connected Semi-Autonomous vehicles do not exist at the moment, as their technologies have yet to be combined. In order for this convergence to take place there must be an increased focus upon traffic safety so that this issue is raised to a level of heightened concern bringing attention and shining a light on the horrific figure of annual traffic fatalities. Both of these technology platforms exist, and in order for this advocacy to move forward and coalesce a champion must emerge to lead the charge. As a society we 
have become so immune to traffic deaths. Some of the technology leaders such as Waymo, GM, or Tesla could easily advocate for this technology combination as its synergistic benefits could very well lead to overwhelming public support for the acquisition of this non-existing product. Corporate entities are so focused upon commercialized fully autonomous vehicles that this myopia misses a great opportunity to save lives now rather than sometime in the future.

\section{My Own Experience}

As an early adopter of connected vehicle technology, I am a volunteer for the CV Tampa Pilot program (see Figure 3). My vehicle includes Level 1-2 Autonomous Vehicle technology in the form of lane assist, emergency braking and parking assist. This low-level A/V technology, in conjunction with the connected vehicle technology provides a suite of safety technology that provides me with the tools necessary to enable greater safety driving.

\section{Conclusions}

While it is impossible to know the future with certainty, the evidence seems very clear that autonomous vehicles will save many lives. Connected vehicles represent a technology that currently exists, but its mass deployment is limited by federal support for the program. Connected vehicle technology pilot programs are limited in scope as their efficacy is being evaluated, and only three cities are currently included in pilot studies. This program should be expanded to include additional cities and be supported by the federal government so that we can do what is necessary in order to bridge the gap until autonomous vehicles are mass adopted.

\section{References}

California Department of Motor Vehicles, Autonomous Vehicle Permits as of 10/18, Retrieved from: https://www.dmv.ca.gov/portal/dmv/detail/pubs/ newsrel/2018/2018 81

Mennie, J., (2019) An examination of autonomous vehicle technology. Muma Business Review 3(17). 195-205. https://doi.org/10.28945/4410

NHSTA, 2017 Traffic Safety Facts Annual Report Tables, Retrieved from: https://cdan.nhtsa.gov/ tsftables/tsfar.htm

Ramsay, M., Self-driving cars could cut down on accidents, study says, 3/5/2015. Wall Street Journal. Retrieved from: https://www.wsj.com/articles/ self-driving-cars-could-cut-down-on-accidentsstudy-says-1425567905

United Nations. Retrieved from: www.who.int/ violence injury prevention/road safety status/2015/en/

Yue, L., Abdel-Aty, M., Wu., Y., \& Wang, L. (2018). Assessment of the safety benefits of vehicles' advanced driver assistance, connectivity and low level automation systems. Accident Analysis and Prevention, 117, 55-64. Retrieved from https://doi-org.flpoly-proxy.flvc.org/10.1016/j. aap.2018.04.002

\section{Review}

This article was accepted under the constructive peer review option. For futher details, see the descriptions at:

http://mumabusinessreview.org/peer-review-options/

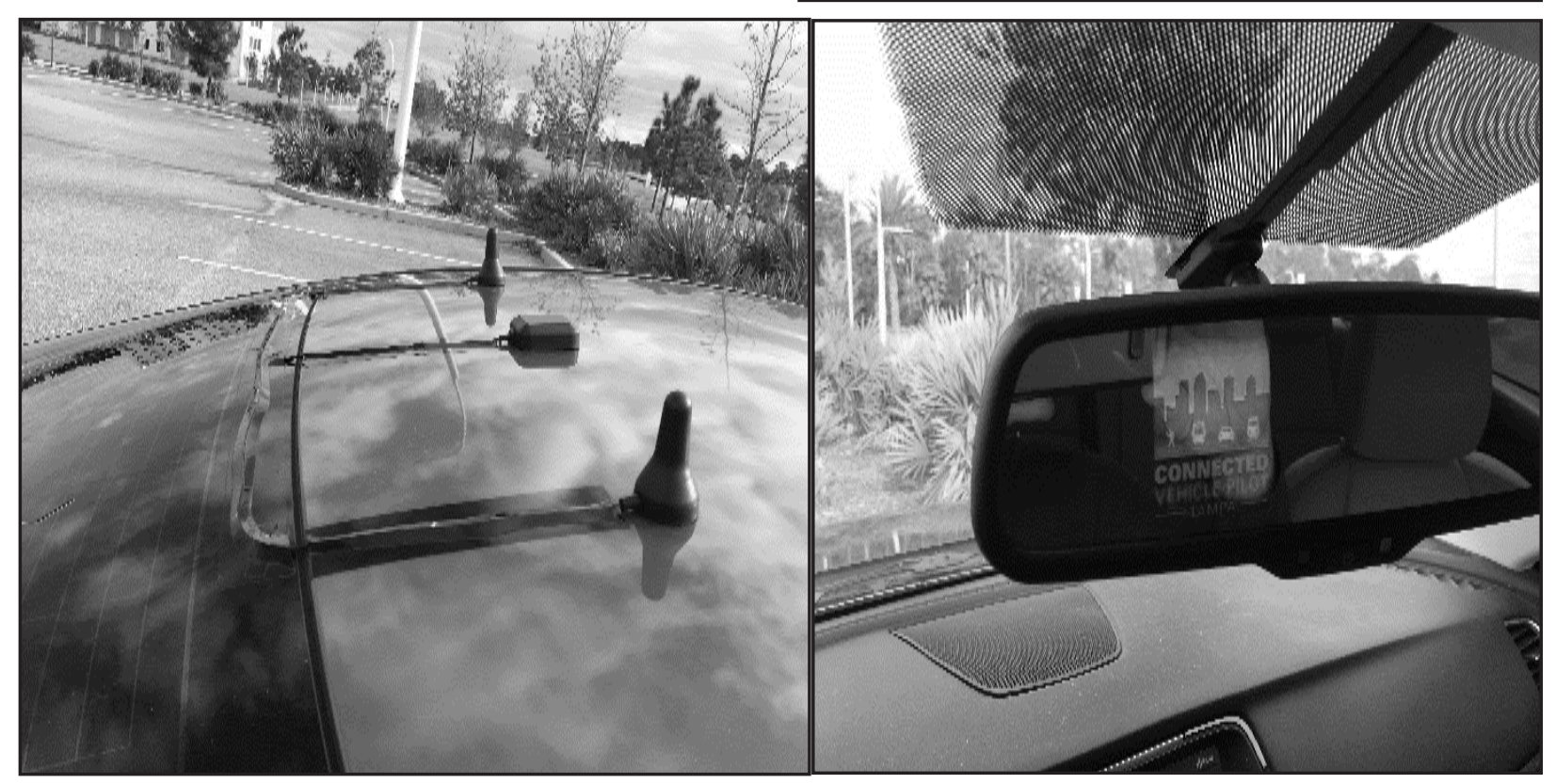

Figure 3: Roof of personal vehicle with connected technology \& enhanced rear-view mirror 


\section{Author}

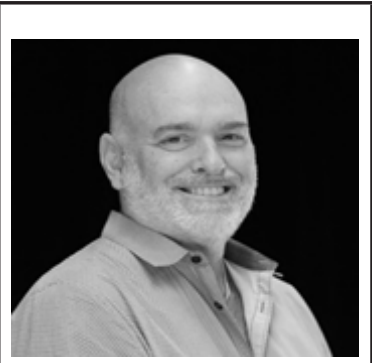

Jim Mennie is an Assistant Professor at Florida Polytechnic University in the Data Science \& Business Analytics Department since 2015. He graduated from the University of South Florida Muma College of Business with his DBA in 2018. He holds an MBA with a concentration in Management from Long Island University, and a Bachelor of Arts degree in Political Science from the State University of New York, College at Oswego. Dr. Mennie has over 25 years experience in improving a broad range of business operations including sales strategies, supplier relations, technology integration, logistics \& supply chain management, project management and increased profitability. 